\title{
USO DE ALGAS MARINAS PARA LA CUANTIFICIÓN FLUOROMÉTRICA DE CLOROFILA-a Y SU APLICACIÓN EN EL DIAGNÓSTICO AMBIENTAL EN AGUAS ESTUARINAS
}

\author{
Químico Fernando Luna Vera ${ }^{1 *}$ \\ Bióloga Marina Tatiana Elina Cardona Forero ${ }^{2 *}$ \\ División de Protección del Medio Marino, Centro Control Contaminación del Pacífico - CCCP \\ Vía El Morro, Capitanía de Puerto, San Andrés de Tumaco, Nariño, Colombia \\ Tél: +57 (2) 72726 37, Fax: +57 (2) 7271180 \\ e-mail: cccp@cccp.org.co \\ fluna@cccp.org.co ${ }^{1 *}$ \\ tcardona@cccp.org.co ${ }^{2 *}$
}

Recibido en marzo de 2004; aceptado en junio de 2004

\section{RESUMEN}

Se evaluó el desempeño de un método fluorométrico para la cuantificación directa de clorofila-a, para esto se utilizó un material con alto contenido de clorofilas, obtenido a partir de algas marinas liofilizadas. Los resultados de la concentración de clorofila-a en las muestras analizadas mostraron alta correlación $(r=0.981)$ con el método espectrofotométrico tricromático, que utiliza las ecuaciones de Jeffrey \& Humphrey (Parsons et al., 1984). Así mismo, la conveniencia del método fluorométrico y del material obtenido a partir de algas marinas se corroboró por medio de un análisis de variables biológicas tales como: abundancia fitoplanctónica y productividad primaria. Obteniéndose resultados que permitieron determinar las condiciones tróficas del área de estudio a un bajo costo.

Palabras claves: Fluorometría, Clorofila-a, Espectrofotometría, Fitoplancton, Productividad Primaria, Condiciones Tróficas.

\section{ABSTRACT}

The perfomance of a direct chlorophyll-a flourometric cuantification method was evaluated. To do this a high chlorophyll content material obtained from marine algae was used. The chlorophyll-a concentration results measured in the analized samples showing high correlation $(r=0.981)$ with those from the tricromatric spectrophotometric method which uses the Jeffrey \& Humphrey (Parsons et al., 1984) equations. Additionally, the flourometric method and the material obtained from marine algae advisability was corroborated by means of biological variable analysis such as: phytoplancton abundance and primary productivity, in this way it was possible to determine the study area trophic conditions at a low cost.

Key words: Fluorometry, Chlorophyll-a, Spectrophotometry, Phytoplankton, Primary Productivity, Trophic Conditions, Method Comparison. 


\section{INTRODUCCIÓN}

La evaluación del contenido de clorofila-a es desde hace varios años un parámetro de importancia para una gran variedad de estudios. Se han realizado, principalmente, trabajos relacionados con la calidad del agua en los que el contenido de clorofila-a en estuarios es utilizado como índice de las condiciones tróficas (EPA, 2003). Así mismo, existe una gran cantidad de información referente al uso de concentraciones de clorofila-a para la estimación de la productividad primaria de ecosistemas marinos (Millán-Núñez et al., 1999; Carpenter et al., 2004); la determinación de clases taxonómicas a partir de espectros de absorción y fluorescencia de clorofila-a en monocultivos de algas (Lynch,1999); el estudio de fenómenos climáticos de interés oceanográfico, como lo son los vientos en el Pacífico Ecuatorial (Rodríguez-Rubio \& Stuardo, 2002), y la evaluación de la actividad fotosintética en corales (Ralph et al., 2002), entre muchos otros.

Los métodos de cuantificación de clorofila-a son, entonces, de vital importancia en todas estas aplicaciones y al respecto se han hecho muchos esfuerzos encaminados a la obtención de métodos rápidos, económicos y eficaces en cuanto a la capacidad del número de muestras o el área de estudio abarcada.

Clásicamente, la cuantificación de clorofila-a se ha realizado por medio de la evaluación espectrofotométrica de extractos en etanol o acetona, registrando su absorbancia a varias longitudes de onda. Este método requiere del uso de ecuaciones, como las de Jeffrey \& Humphrey o Lorenzen, las cuales intentan discriminar la señal producida por la clorofila-b, los feopigmentos y la turbidez de la muestra (Parsons et al., 1984; EPA, 1997). A pesar de que este método es aceptado como el método de referencia, tiene algunas desventajas como su gran sensibilidad a la turbidez de la muestra y los esfuerzos que exige mantener constante el volumen de extracción.

La utilización de High Perfomance Liquid Chromatography, HPLC, como método de separación y cuantificación de clorofila-a ha sido también investigada, arrojando resultados aceptables cuando se ha comparado con la espectrofotometría (Louda \& Monghkonsri, 2004). No obstante, a pesar de sus ventajas relacionadas, principalmente, con la identificación inequívoca de clorofila-a, es un método poco práctico para ser utilizado como rutinario, debido a su elevado costo y tiempo de operación por muestra analizada, ya que se obtienen tiempos de retención de entre 15 y 20 minutos ( $\mathrm{Li}$ et al., 2002).

Adicionalmente, se han hecho varios estudios en la cuantificación de clorofilas usando imágenes satelitales, provenientes de sensores remotos como IRS-P4 (Dey \& Singh, 2003) y SeaWIFS (Moore \& Abbott, 2000); métodos que tienen la ventaja de cubrir amplias áreas de estudio, sin la recolección de muestras o desplazamiento a campo.

De otra parte, el fenómeno de fluorescencia presentado por la clorofila-a ha sido aprovechado, ampliamente, para generar instrumentos y técnicas fluorométricas como métodos de cuantificación. La fluorometría tiene ventajas interesantes sobre los métodos clásicos debido, especialmente, al corto tiempo de análisis, los bajos límites de detección y su gran sensibilidad la cual llega a ser más de mil veces que la de los métodos espectrofotométricos (Turner Desing, 2004); así mismo, ha llegado a ser ampliamente aplicada por su facilidad de utilización en determinaciones in situ (Ostrowska, et al., 2000).

Sin embargo, la fluorometría sigue considerándose un método indirecto, por tanto es indispensable su calibración con un método de referencia como la espectrofotometría, a menos que se cuente con un material de referencia de clorofila-a que permita la construcción de curvas de calibración. Dicho material tiene, por lo general, un alto costo.

El presente trabajo estudió la cuantificación fluorométrica directa de clorofila-a in vitro, utilizando para ello un material con alto contenido de clorofila-a construido en el laboratorio a partir de algas marinas, el cual tiene como inmensa ventaja su bajo costo.

La validez del método se evaluó por comparación con el método espectrofotométrico, con datos históricos de la región estudiada; así como con las relaciones obtenidas con otras variables biológicas y climáticas que intervienen en la dinámica de clorofilas en sistemas estuarinos, como la abundancia fitoplanctónica y las precipitaciones. 


\section{ÁREA DE ESTUDIO}

La recolección de muestras para el análisis de clorofila-a se llevó a cabo en 21 estaciones ubicadas en la bahía de Tumaco, la cual se encuentra en el extremo sur del litoral Pacífico colombiano (Fig. 1), entre las latitudes $1^{\circ} 45^{\prime} \mathrm{N}$ y $2^{\circ} 00^{\prime} \mathrm{N}$ y las longitudes $78^{\circ} 30^{\prime} \mathrm{N}$ y $78^{\circ} 45^{\prime} \mathrm{O}$, comprende un área aproximada de $350 \mathrm{Km}^{2}$ y profundidades entre 0 y $50 \mathrm{~m}$ (Tejada, 2002). Presenta un régimen mareal semidiurno, con amplitud máxima de $4 \mathrm{~m}$.

La bahía de Tumaco se caracteriza por tener un clima tropical húmedo. Las lluvias son de tipo monomodal, considerándose el invierno en el primer semestre y la época de verano el resto del año (CCCP, 2002). Cuenta con ríos cortos pero caudalosos, cuyas principales cuencas las constituyen los ríos Rosario, Mexicano, Tablones, Gualajo, Colorado y Changuií, entre otras. Esta configuración hace que la bahía se comporte como un sistema estuarino.

\section{METODOLOGÍA}

\section{Toma y preservación de muestras}

Se colectaron 30 muestras de agua, a través de la red de estaciones de muestreo (Fig. 1) distribuidas en la bahía de Tumaco. Las muestras fueron tomadas con botella Niskin en dos condiciones de marea (alta y baja), durante el mismo día y transportadas en bidones plásticos de polietileno hasta el laboratorio.

Para cada muestra el fitoplancton se concentró por medio de la filtración al vacío de $700 \mathrm{~mL}$ de agua sobre filtros de celulosa de $0.45 \mu \mathrm{m}$ de tamaño de poro (marca Schleicher \& Schuell). Los filtros fueron guardados luego en sobres de papel aluminio y almacenados a $-10^{\circ} \mathrm{C}$ hasta su análisis.

\section{Preparación del material de algas marinas}

Se colectaron algas clorophytas de la familia cloroficeae atrapadas entre el manglar (Rizophora mangle) presente en una zona intermareal del área de estudio. El material vegetal colectado fue liberado de pequeños residuos y organismos, y posteriormente lavado en varias ocasiones con agua destilada. La humedad superficial fue retirada utilizando papel absorbente.

Con el objeto de producir un material estable, libre de humedad y de fácil manipulación las algas obtenidas fueron liofilizadas a $-50^{\circ} \mathrm{C}$ y $1.33 \times 10^{-5} \mathrm{mBar}$ durante tres horas en un equipo Freeze Dry System modelo 77510 de Labconco. El material obtenido fue tamizado en un tamiz \# 40 de $425 \mu \mathrm{m}$, empacado en un vial, protegido de la luz con papel aluminio y almacenado bajo refrigeración hasta su uso para la preparación de curvas de calibración.

\section{Calibración y parámetros de desempeño}

Los espectros y medidas de concentración se realizaron en un espectrofluorofotómetro Shimadzu modelo RF-5301PC, controlado por el software Personal Fluorescence Software V 1.4.

Se determinaron las condiciones instrumentales, tales como el ancho de banda y las longitudes de excitación (EX) y emisión (EM), que produjeran la más alta señal instrumental para un extracto en acetona al $90 \%$ de $10 \mathrm{mg}$ de algas clorophytas. Para ello se registraron espectros de emisión en el rango de 350 a $700 \mathrm{~nm}$ para los cuales se utilizó como longitudes de onda de excitación 380, 400, 430, 480 y $520 \mathrm{~nm}$.

La concentración de clorofila-a en el extracto de algas se determinó por medio de la aplicación del método espectrofotométrico y las ecuaciones de Jeffrey \& Humphrey (Parsons et al., 1984) a extractos obtenidos sobre cantidades variables del material vegetal, usando un espectrofotómetro UVVis Perkin-Elmer Lamda 3.

Se construyeron siete curvas de calibración, cada una con cinco puntos: 1.30, 0.650, 0.325, 0.163, y $0.080 \mathrm{mg} / \mathrm{L}$, obtenidos por medio de diluciones sucesivas $1: 2$ a partir de la solución concentrada de $1.3 \mathrm{mg} / \mathrm{L}$ de clorofila-a y un volumen total de 10 $\mathrm{mL}$. Se obtuvo una única curva de calibración promediando los siete valores de intensidad para cada punto de calibración y utilizando una regresión por mínimos cuadrados. Se midieron en diez ocasiones patrones de 0.650 y $0.085 \mathrm{mg} / \mathrm{L}$, con el objeto de evaluar la precisión del sistema. Adicionalmente, se midieron diez blancos de reactivos (solución de extracción) para determinar el límite de detección.

Los extractos de $1.3 \mathrm{mg} / \mathrm{L}$ fueron preparados tomando $20+/-0.2 \mathrm{mg}$ del material de algas en un tubo de ensayo, en el cual se adicionó $10 \mathrm{~mL}$ de acetona (Pestanal, Riedel - de Haën) al 90\% en 
agua destilada. A continuación el tubo se agitó vigorosamente por cinco minutos. Se dejó decantar el residuo y, finalmente, se transvasó el sobrenadante a otro tubo limpio y seco.

\section{Análisis fluorométrico de muestras de fitoplancton}

Cada uno de los filtros preservados bajo refrigeración fue disuelto por agitación en $10 \mathrm{~mL}$ de acetona al $90 \%$ dentro de tubos de ensayo. A continuación fueron centrifugados y el sobrenadante fue transvasado directamente a la celda de medida del espectrofluorofotómetro e interpolada la lectura de intensidad directamente en la curva de calibración establecida. Adicionalmente, los mismos extractos fueron analizados el mismo día por espectrofotometría, con el objeto de comparar los dos métodos.

\section{Análisis complementarios}

Se tomaron separadamente aproximadamente $500 \mathrm{~mL}$ de muestra en cada estación para llevar a cabo el análisis de abundancia fitoplanctónica por medio de conteo celular en un microscopio óptico (Leica Galen III), luego de fijar cada muestra con formol al $10 \%$.

\section{RESULTADOS Y DISCUSIÓN}

\section{Material de algas marinas}

Se obtuvieron $451 \mathrm{mg}$ de material vegetal, el cual se usó para la construcción de curvas de calibración. Su caracterización, por medio del análisis espectro-fotométrico de la concentración de clorofila-a (Clo-a) sobre cantidades variables de algas liofilizadas (Tabla 1), mostró que dicho material tiene una concentración de $0.634+1$ $0.041 \mathrm{mg}$ Clo- a $/ \mathrm{g}$ de material vegetal $(P=0.05)$.

\section{Consideraciones Analíticas}

La longitud de onda de emisión de los extractos acetónicos se registró a $667.0 \mathrm{~nm}$, usando un ancho de banda de $5 \mathrm{~nm}$, y la intensidad de dicha emisión resultó ser dependiente de la longitud de onda excitación (EX) utilizada (Fig. 2), presentando una intensidad máxima a $430 \mathrm{~nm}$.
La curva de calibración fluorométrica de Clo-a presentó linealidad hasta $0.650 \mathrm{mg} / \mathrm{L}$, soluciones más concentradas tornaron la ecuación de calibración en polinomios de segundo orden (Fig. 3). La ecuación de la recta de calibración fue $Y=365.9 X+6.8641$, con un coeficiente de correlación $r^{2}=0.9962$ y un intercepto significativamente diferente de cero $(P$ $=0.05)$. La sensibilidad del método fluorométrico fue de 366 unidades de intensidad/(mg/L) de Clo-a.

La prueba de precisión mostró coeficientes de variación (CV) de 0.42 y $1.17 \%$ para 0.650 y $0.085 \mathrm{mg} / \mathrm{L}$, respectivamente.

El límite de detección, con incertidumbre del $95 \%$, es de $0.16 \mu \mathrm{g} / \mathrm{L}$ Clo-a. Este valor resulta menor en un orden de magnitud al reportado $(5.8 \mu \mathrm{g} / \mathrm{L})$ para Cromatografía Líquida de Alta Presión, HPLC (por sus siglas en inglés) (Li et al., 2002).

\section{Comparación de Métodos}

Las muestras colectadas en las 21 estaciones de muestreo se analizaron por los métodos espectrofotométrico y fluorométrico (Fig. 4) en el cual se observa una línea de tendencia con ecuación $Y=1.058 X+0.2105$, que muestra la existencia de concordancia entre los resultados obtenidos por el método fluorométrico, en el que se usó el material de algas clorophytas, y el método de referencia (espectrofotométrico), con un error relativo de la pendiente con respecto al valor esperado $(m=1)$ de $5.6 \%$. La correlación entre los dos métodos evidenciado es $r=0.981$, la cual resultó significativa a $P=0.01$.

El análisis de la distribución de las diferencias de concentración entre métodos (Fig. 5) no muestra tendencias que permitan evidenciar la presencia de errores sistemáticos. Sin embargo, a pesar de que no se evidencian diferencias proporcionales, es claro que para la mayoría de las muestras el contenido de Clo-a, calculado por el método fluorométrico, es menor al obtenido por espectrofotometría.

\section{Verificación de resultados}

Cuando se evalúan parámetros fisicoquímicos con el objeto de estimar el estado salud de un ecosistema hay que tener en cuenta que estos dependen de factores bióticos como la diversidad y abundancia de 
individuos, número de taxas, entre otros; por tanto no es suficiente con tener datos puntuales. En lugar de esto se utilizan rangos de variación con los cuales es posible establecer criterios individuales para cada tipo de ecosistema.

. Es común, entonces, evaluar el comportamiento de la variable de estudio, como es el caso de la clorofila, mediante series de espacio y tiempo, para así establecer cambios en los valores normales observados.

Con el método de fluorometría estudiado se evaluó el contenido de clorofila-a superficial $(0-0.5 \mathrm{~m}$ de profundidad) en las estaciones de muestreo distribuidas en la bahía de Tumaco, en tres épocas del año 2003, las cuales coinciden con los períodos de lluvia (540.6 mm/ $/ \mathrm{m}^{2}$ en marzo), el período seco ( $90.3 \mathrm{~mm} / \mathrm{m}^{2}$ en julio) y el período de transición ( $221.6 \mathrm{~mm} / \mathrm{m}^{2}$ en noviembre).

La Tabla 2 muestra los valores promedio de clorofila-a encontrados para las tres épocas de muestreo en dos estados de marea. Se observa que las concentraciones de clorofila-a son influenciadas por el régimen de marea, encontrándose variaciones entre estados de marea de 30.6, 29.0 y $37.9 \%$ en la concentración de Clo-a para marzo, julio y noviembre, respectivamente.

Adicionalmente, se observa que las concentraciones de Clo-a promedio entre mareas están altamente correlacionadas $\left(r^{2}=0.9997\right)$ con la precipitación (Fig. 6), lo cual es un resultado esperado ya que la lluvia mejora las condiciones ambientales del medio, aumentando el aporte de nutrientes por parte de los ríos que desembocan en la región de estudio (fosfatos y nitratos), los cuales son esenciales para el crecimiento de las microalgas (Margalef, 1982).

Por otra parte, la bahía de Tumaco se caracteriza por un intercambio continuo de agua de características oceánicas con agua de ríos, lo que hace que se comporte como un sistema estuarino de alta productividad, esto se corrobora con los resultados de Clo-a arrojados por el método fluorométrico, calibrado con el material de algas clorophytas estudiado.

Se realizó con anterioridad a este trabajo la determinación de la productividad primaria bruta por medio del método de evolución de oxígeno (Parsons et al., 1984) en tres estaciones representativas de la bahía. En esa ocasión se encontró que el área con mayor productividad (195.26 $\mathrm{mgO}_{2} / \mathrm{m}^{3} \mathrm{~h} \mathrm{ppb}$ ) es la ubicada en la zona de influencia de los ríos (Rosario, Mexicano, Tablones). Este resultado fue confirmado por el método fluorométrico ya que se encontró que la más alta productividad de toda la red de estaciones, medida como contenido de clorofila-a, resultó en el área de los ríos $(23 \mu \mathrm{g} / \mathrm{L})$.

Más interesante aún resultó el hecho que las mayores concentraciones de clorofila-a coinciden con la mayor abundancia de microalgas la cual se agrupa entre rangos de variación de 56 y $165 \mathrm{cel} / \mathrm{mL}$. Presentándose el mayor número de células de la comunidad fitoplanctónica en la zona influenciada por la entrada de aguas continentales, como se aprecia en la Figura 7.

Estos hechos verifican que los resultados obtenidos por el método fluorométrico son congruentes con los esperados para el sistema de estudio, así como con las conclusiones arrojadas por métodos alternos (espectrofometría) y por tanto el método es apto para la vigilancia y el diagnóstico ambiental.

Los resultados en las concentraciones de clorofila-a para el período de muestreo (2003) muestran rangos de variación de $0.2 \mathrm{a} 18 \mu \mathrm{g} / \mathrm{L}$ en marea alta y de 0.6 a $11.05 \mu \mathrm{g} / \mathrm{L}$ en marea baja, valores que indican que el cuerpo de agua funciona adecuadamente en la producción de biomasa fitoplanctónica, por lo que se considera como un sistema de condiciones mesotróficas.

\section{CONCLUSIONES}

El método fluorométrico para determinación de clorofila-a en aguas marinas, el cual usa un material construido en laboratorio, a partir de algas marinas clorophytas, demostró tener un buen desempeño, evidenciado en parámetros analíticos como linealidad y repetibilidad. Asimismo demostró, por medio de la corroboración con el comportamiento esperado para la zona de estudio, que puede ser utilizado para diagnóstico ambiental.

Intrínsecamente, se evidenció que para la calibración del espectrofluorómetro utilizado no es necesaria la utilización de un patrón de referencia de clorofila-a de alto costo, sino que para fines ambientales es posible utilizar material vegetal cuyo 
contenido de Clorofila-a sea alto y esté especificado.

\section{REFERENCIAS BIBLIOGRÁFICAS}

Carpenter, E., A. Subramaniam and D. Capone. 2004. Biomass and Primary Productivity of the Cyanobacterium Trichodesmium spp. In the Tropical Atlantic Ocean, Deep-sea research I 51, pp 173-203.

Centro Control Contaminación del Pacífico. 2002. Compilación Oceanográfica de la Cuenca Pacífica Colombiana. Centro Control Contaminación del Pacífico. San Andrés de Tumaco : CCCP, Colombia, 107 pp.

Dey, S. \& R. Singh. 2003. Comparison of Chlorophyll Distributions in the Northeastern Arabian Sea and Southern Bay of Begal Using IRS-P4 Ocean Color Monitor Data, Remote Sensing of Environment, 85, pp. $424-428$.

Environmental Protection Agency. 1997. Lake Michigan Mass Balance Methods Compendium, Volumen 4, Capítulo 3. EPA. [en-línea]. URL: http:/ /www.epa.gov/ghpo//mmb/methods/ methd150.pdf. [Consulta: 16 de febrero 2004].

Environmental Protection Agency. 2003. Chesapeake Bay Program Office. Ambient Water Quality Criteria for Dissolved Oxygen, Water Clarity and Chlorophyll- a for the Chesapeake Bay and it's tidal tributaries. EPA. Annapolis Mariland, pp. 101-137.

Li, H., G. Gong and T. Hsiung. 2002. Phytoplankton Pigment Analysis by HPLC ans its Application in Algal Communiy Investigations, Bot. Bull. Acad. Sin, 43, pp. 283-290.

Louda, J. \& P. Monghkonsri. 2004. Comparison of Spectrophotometric Estimates of Chlorophylls-a, $-b,-c$ and 'pheopigments' in Florida Bay Seston with that Obtained by High Performance Liquid Chromatography-Photodiode Array Analyses, Florida Scientist (in press).

Lynch, P. 1999. Using Bio-optical Taxonamy to Determine or Distinguish between Problematic Phytoplankton Species. PhD. Thesis. University College Dublin.

Margalef, R. 1982. Ecología, Ediciones Omega S.A., España. 435 pp.

Millán-Núñez. R, E. Santamaría del Ángel, R. Cajal-Medrano and O. Barocio-León. 1999. The
Colorado River Delta: a High Primary Productivity Ecosystem. Ciencias Marinas, 25 (4), pp. 509-524.

Moore, J. \& M. Abbott. 2000. Phytoplankton Chlorophyll Distributions and Primary Production in the Southern Ocean. Journal of Geophysical Research, Vol. 105, No. C12, pp. 28, 709-28, 722.

Ostrowska, M., R. Majchrowski, D. Matorin and B. Wozniak. 2000. Variability of the Specific Fluorescence of Chlorophyll in the Ocean. Par 1. Theory of classical in situ chlorophyll fluorometry, Oceanologia 42 (2), pp. 203-219.

Parsons T., Y. Maila and C. Lalli. 1984. A Manual of Chemical and Biological Methods for Seawater Analysis, Pergamon, 173 pp.

Ralph, P., R. Gademan R, A. Larkum and T. Kuilhl. 2002. Spatial Heterogenity in Active Chlorophyll Fluorescence and PSII Activity of Coral Tissues. Marine Biology. 141: pp. 639-646.

Rodríguez-Rubio E. \& J. Stuardo. 2002. Variability of the Photosynthetic Pigments in the Colombian Pacific Ocean and its Relation Ship With the Wind Field Using ADEO-I Data, Proc. Indian. Acad. Sci (earth planet science), 111, No. 3, pp 227-236.

Tejada, C. 2002. Clima Marítimo de la Bahía de Tumaco, un Caso de Aplicación del Sistema Integrado de Zona Costera para Colombia. Cantabria, 44 pp. Tesis de maestría (MSc en Ciencias Tecnológicas para la Gestión de la Costa). Universidad de Cantabria. Grupo de Ingeniería Oceanográfica y de Costas.

Turner Designs. 2004. Support Application Notes, Fluorometric Facts Bulletin 101. [en-línea]. URL: http:/ /www.turnerdesigns.com/t2/doc/appnotes/ 998_5101.html. [Consulta: 16 de enero 2004]. 
FIGURAS Y TABLAS

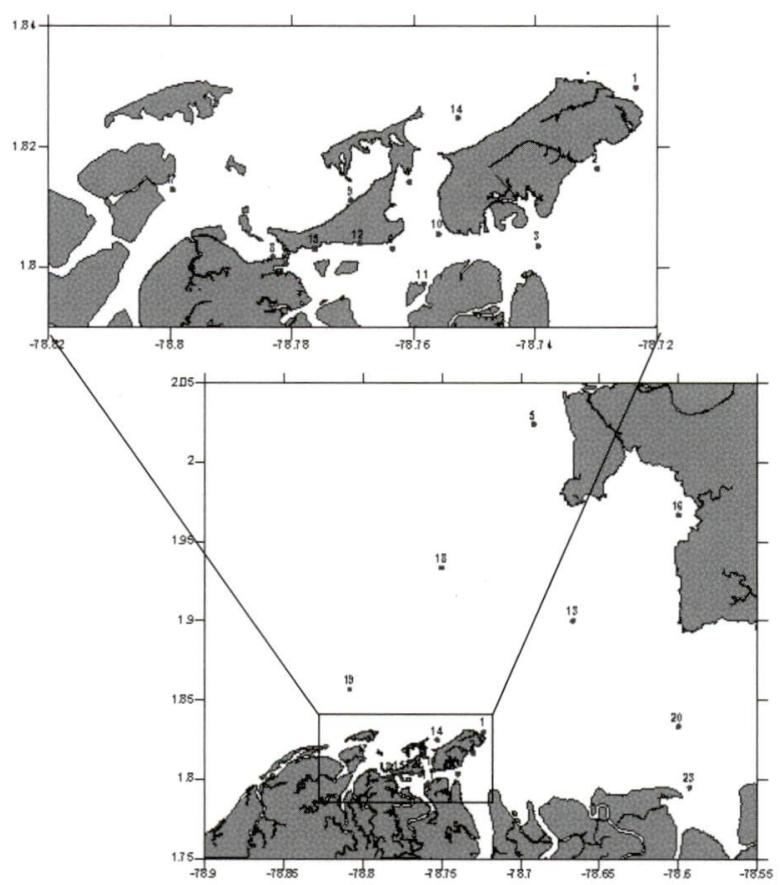

Figura 1. Área de estudio, bahía de Tumaco.

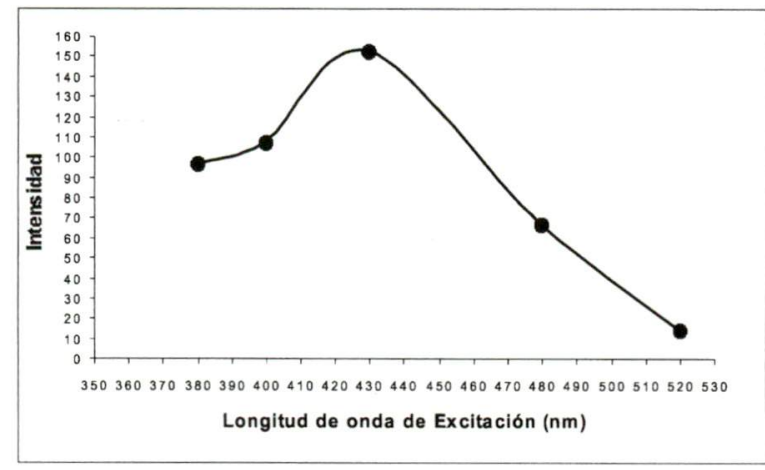

Figura 2. Variación de la intensidad de emisión a $667 \mathrm{~nm}$, con respecto a la longitud de onda de excitación.

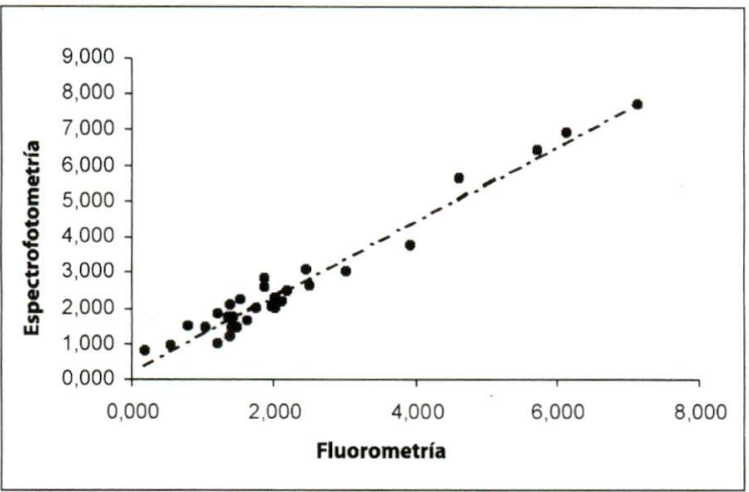

Figura 4. Comparación de resultados obtenidos por espectrofotometría y fluorometría.

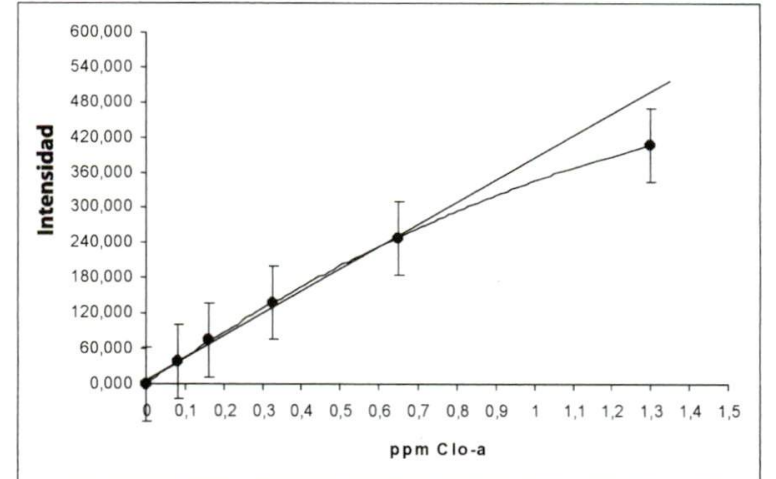

Figura 3. Comportamiento de la concentración de Clo-a vs. la intensidad de fluorescencia.

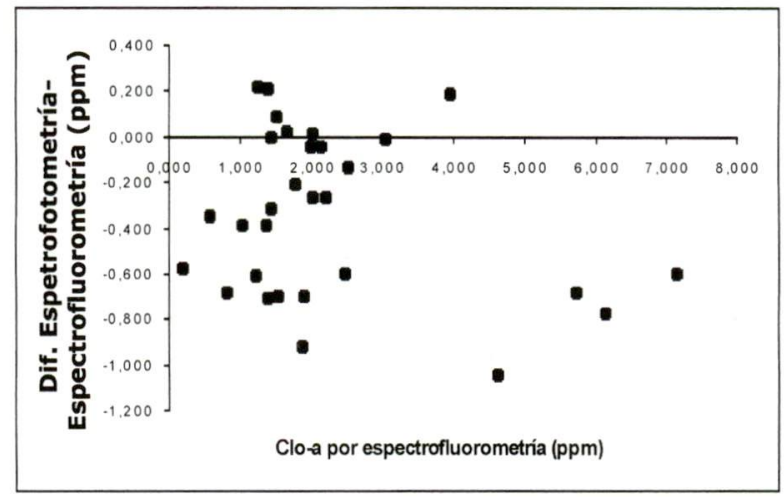

Figura 5. Diferencias en concentación de Clo-a entre los métodos estudiados. 


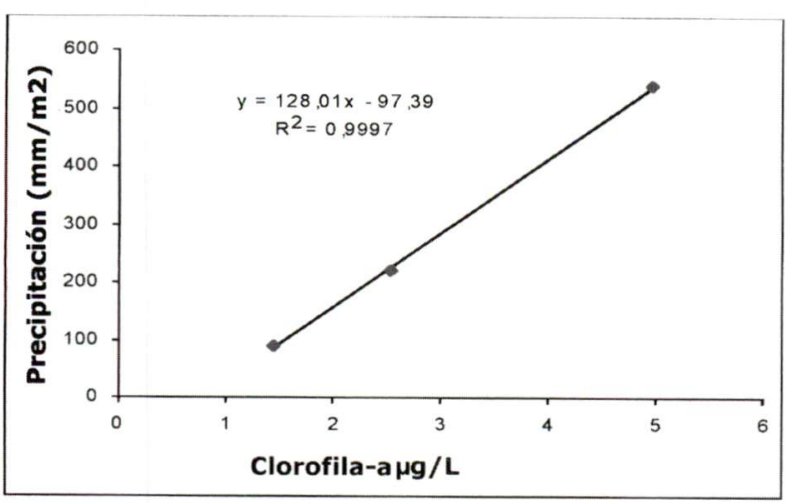

Figura 6. Variación de clorofila-a promedio con la precipitación en la bahía de Tumaco para el año 2003.

Tabla1. Valores de masa y concentración usados para caracterización de marerial de referencia.

\begin{tabular}{|c|c|}
\hline Masa de algas (g) & Concentración de Clo-a ( $\mathbf{m g} / \mathbf{g})$ \\
\hline 0.01997 & 698.5 \\
\hline 0.02018 & 706.7 \\
\hline 0.02010 & 587.5 \\
\hline 0.02026 & 634.3 \\
\hline 0.01990 & 620.7 \\
\hline 0.02026 & 654.9 \\
\hline 0.01998 & 538.1 \\
\hline
\end{tabular}

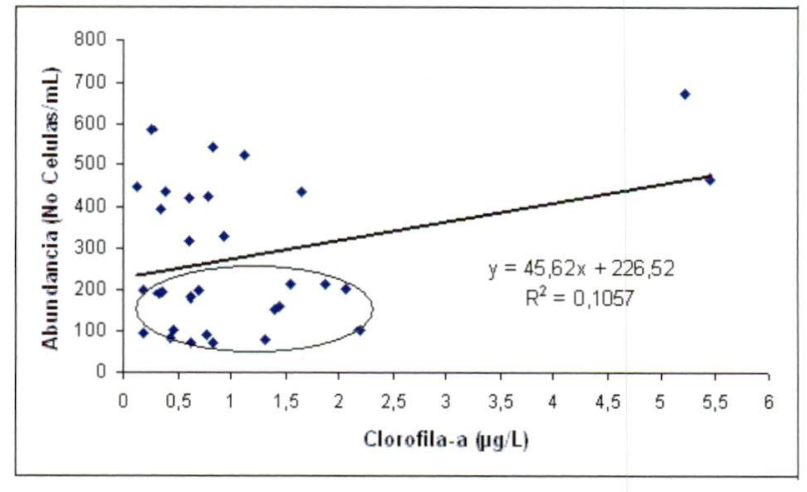

Figura 7. Relación de abundancia fitoplanctónica.

Tabla 2. Clorofila-a promedio en dos estados de marea, para tres períodos de muestreo en la bahía de Tumaco.

\begin{tabular}{|c|c|c|}
\hline & $\begin{array}{c}\text { Marea Alta } \\
\text { (ppb) }\end{array}$ & $\begin{array}{c}\text { Marea Baja } \\
\text { (ppb) }\end{array}$ \\
\hline Marzo & 5.73 & 4.21 \\
\hline Julio & 1.23 & 1.65 \\
\hline Noviembre & 3.01 & 2.05 \\
\hline
\end{tabular}

